\title{
Observation of biexcitons in monolayer $\mathrm{WSe}_{2}$
}

\author{
Yumeng You ${ }^{1,2 \dagger}$, Xiao-Xiao Zhang ${ }^{2 \dagger}$, Timothy C. Berkelbach ${ }^{3}$, Mark S. Hybertsen ${ }^{4}$, David R. Reichman ${ }^{3}$ \\ and Tony F. Heinz ${ }^{2 \star *}$
}

\begin{abstract}
Transition metal dichalcogenide (TMDC) crystals exhibit new emergent properties at monolayer thickness ${ }^{1,2}$, notably strong many-body effects mediated by Coulomb interactions ${ }^{3-6}$. A manifestation of these many-body interactions is the formation of excitons, bound electron-hole pairs, but higher-order excitonic states are also possible. Here we demonstrate the existence of four-body, biexciton states in monolayer $\mathrm{WSe}_{2}$. The biexciton is identified as a sharply defined state in photoluminescence at high exciton density. Its binding energy of $52 \mathrm{meV}$ is more than an order of magnitude greater than that found in conventional quantum-well structures ${ }^{7}$. A variational calculation of the biexciton state reveals that the high binding energy arises not only from strong carrier confinement, but also from reduced and non-local dielectric screening. These results open the way for the creation of new correlated excitonic states linking the degenerate valleys in TMDC crystals, as well as more complex many-body states such as exciton condensates or the recently reported dropletons ${ }^{8}$.
\end{abstract}

TMDC crystals, including $\mathrm{MoS}_{2}, \mathrm{MoSe}_{2}, \mathrm{WS}_{2}$ and $\mathrm{WSe}_{2}$, are semiconductors that form layered structures with a plane of hexagonal metal atoms surrounded by two planes of chalcogen atoms in trigonal prismatic coordination. At monolayer thickness, these crystals exhibit direct band gaps at the $\mathrm{K}$ and $\mathrm{K}^{\prime}$ points in the Brillouin zone $^{1,2}$, and recent studies have revealed the possibility of selectively accessing the $\mathrm{K}$ or $\mathrm{K}^{\prime}$ valley through the use of circularly polarized light ${ }^{9-12}$, as well as the existence of an associated valley Hall effect ${ }^{13}$. Importantly, many-body Coulomb interactions in these monolayer TMDC crystals have been found to be particularly strong. This leads to excitonic optical transitions in the materials, with exciton binding energies of several hundred $\mathrm{meV}$ (refs 3-6). In the presence of free charges, stable charged excitons (trions) have also been identified and exhibit binding energies of tens of $\mathrm{meV}$ (refs 12,14-16).

In view of the prominence of these two- and three-body excitonic states, it is natural to ask whether two-dimensional (2D) TMDC materials, just as for the much-studied zero- and one-dimensional nanostructures ${ }^{7,17-21}$, also support the formation of stable biexcitons ${ }^{22,23}$. Here we demonstrate the presence of biexcitons in monolayer $\mathrm{WSe}_{2}$ through the discovery of a sharp new emission peak under pulsed laser excitation. We further probe the properties of the biexciton state through measurements of its ultrafast dynamics, valley polarization and thermal stability. We establish a biexciton binding energy of $52 \mathrm{meV}$. This unusually high binding energy is compatible with results of a variational analysis of biexcitonic states performed using a non-locally screened Coulomb potential to describe the interactions of charges in the atomically thin 2D material.
To identify the biexciton states, we have studied the photoluminescence (PL) under ultrafast pump radiation at different fluences, producing different exciton densities. We compare the PL spectra for a $\mathrm{WSe}_{2}$ monolayer at a temperature of $50 \mathrm{~K}$ for two excitation fluences (Fig. 1a). The higher applied fluence of $12 \mu \mathrm{J} \mathrm{cm}^{-2}$ yields an exciton density up to $5 \times 10^{11} \mathrm{~cm}^{-2}$ (see Methods). Several distinct emission features are observed in the PL spectra, most of which have been identified in previous investigations at low excitation density ${ }^{12}$. In particular, the peak at $1.74 \mathrm{eV}$ arises from emission from neutral excitons $(\mathrm{X})$ and the peak at $1.71 \mathrm{eV}$ from negative trions $\left(\mathrm{X}^{-}\right)$. At lower photon energies, we see additional emission features, which we label as $\mathrm{P}_{0}$ to $\mathrm{P}_{3}$. Features $\mathrm{P}_{1}$ to $\mathrm{P}_{3}$ grow more slowly than exciton emission with increasing pump fluence, exhibiting sublinear fluence dependence. We assign $\mathrm{P}_{1}$ to $\mathrm{P}_{3}$ to emission from bound excitons at defect sites ${ }^{24}$, in agreement with previous PL studies ${ }^{12,25}$.

The behaviour of peak $\mathrm{P}_{0}$ at $1.68 \mathrm{eV}$ is, however, different from that of the other lower-energy features. This feature grows superlinearly with fluence. To probe the properties of emission feature $\mathrm{P}_{0}$ in more detail, we have measured $\mathrm{PL}$ spectra at $10 \mathrm{~K}$ over a wide range of excitation fluence (Fig. 1b). At high excitation densities, feature $\mathrm{P}_{0}$ emerges as the strongest emission channel.

For a quantitative analysis of the different emission states, we fit the emission spectra to a form with one symmetrical feature for each of the $\mathrm{P}_{0}-\mathrm{P}_{3}$, $\mathrm{X}$ and $\mathrm{X}^{-}$peaks (see Supplementary Information). We obtain (Fig. 1b) a good representation of the observed spectra at different excitation fluences solely by varying the intensity of the emission peaks; the energies and linewidths of the features are unchanged with fluence.

Our primary interest lies in understanding the superlinear behaviour of the $\mathrm{P}_{0}$ feature. To minimize the influence of defect states, we analyse emission from the $\mathrm{P}_{0}$ feature in terms of the strength of the neutral exciton emission. As the emission times of $\mathrm{X}, \mathrm{X}^{-}$and $\mathrm{P}_{0}$ show no significant variation with fluence (see below), we associate the strengths of the PL features with the population of the corresponding emitting species. Figure $1 \mathrm{c}$ shows the emission strength $I_{\mathrm{P}_{0}}$ of the $\mathrm{P}_{0}$ species as a function of the emission intensity $I_{\mathrm{X}}$ of the neutral exciton. The $\mathrm{P}_{0}$ data can be described adequately by a power-law relation of the form $I_{\mathrm{P}_{0}} \propto I_{\mathrm{X}}{ }^{\alpha}$, with $\alpha=1.39$. (See Supplementary Information for further discussion of the fluence dependence of the different species.)

On the basis of the superlinear strength of emission from the $\mathrm{P}_{0}$ species with respect to $\mathrm{X}$ emission, we identify this feature as arising from a biexciton (XX) state. Under conditions of full thermal equilibrium (neglecting any possible rise in temperature with increased excitation fluences), we would expect a quadratic

\footnotetext{
${ }^{1}$ Ordered Matter Science Research Center, Southeast University, Nanjing 211189, China. ${ }^{2}$ Departments of Physics and Electrical Engineering, Columbia University, 538 West 120th St., New York, New York 10027, USA. ${ }^{3}$ Department of Chemistry, Columbia University, 3000 Broadway, New York, New York 10027, USA. ${ }^{4}$ Center for Functional Nanomaterials, Brookhaven National Laboratory, Upton, New York 11973-5000, USA. †These authors contributed equally to this work. tPresent addresses: Department of Applied Physics, Stanford University, Stanford, California 94305, USA; SLAC National Accelerator Laboratory, 2575 Sand Hill Rd., Menlo Park, California 94025, USA. *e-mail: tony.heinz@stanford.edu
} 


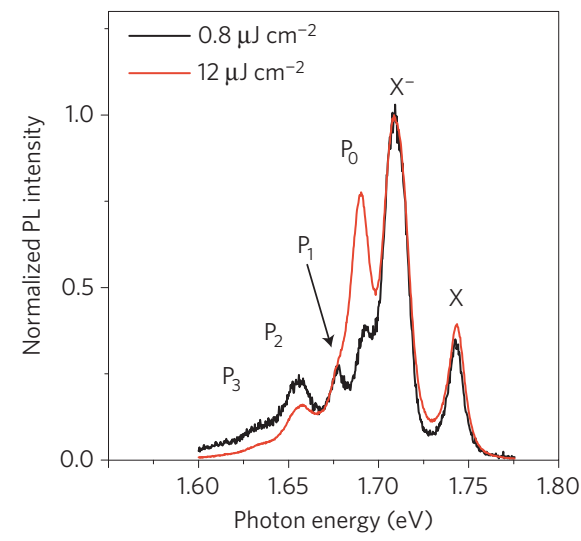

b

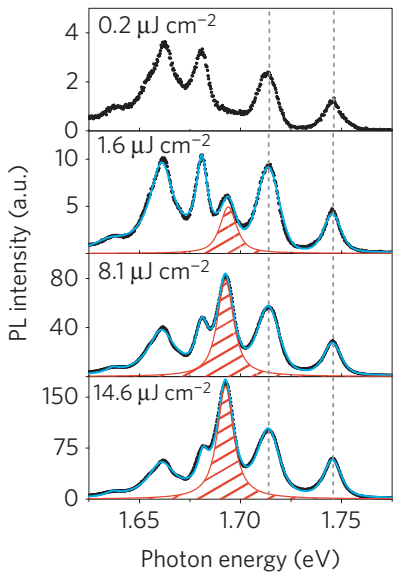

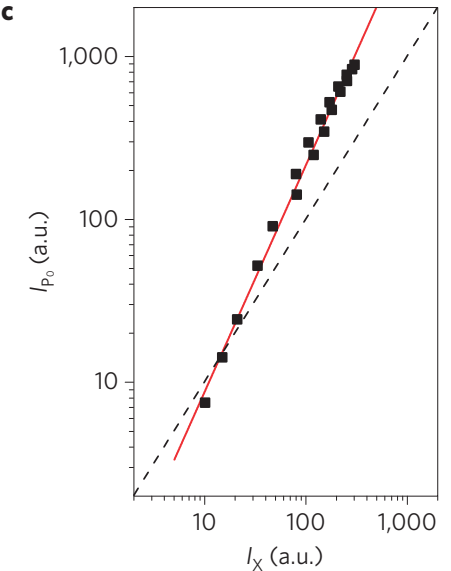

Figure 1 | Photoluminescence spectra and intensities of monolayer $\mathbf{W S e} \mathrm{e}_{2}$ for different pump fluences. a, PL spectra at $50 \mathrm{~K}$ for pulsed excitation under applied fluences of $0.8 \mu \mathrm{J} \mathrm{cm}^{-2}$ and $12 \mu \mathrm{J} \mathrm{cm}{ }^{-2}$. The spectra are normalized to yield the same emission strength for the neutral exciton. $\mathbf{b}, \mathrm{PL}$ spectra and spectral fitting at $10 \mathrm{~K}$ for the indicated applied fluences. The major emission features are the same as those in $\mathbf{a}$. The vertical grey lines show that there is no peak shift of the features with fluence. The black dots are experimental data, and the solid blue lines are based on a fit with one feature for each peak, where only the peak amplitudes are changed for the different spectra. The component of the $P_{0}$ peak is shown separately in red. c, Logarithmic plot of the $P_{0}$ emission strength, $I_{P_{0}}$, as a function of the exciton emission strength $I_{X}$. The red line is a power-law fit: $I_{P_{0}} \propto I_{X}{ }^{\alpha}$, with $\alpha=1.39$. For comparison, a linear relation is shown as a dashed black line.

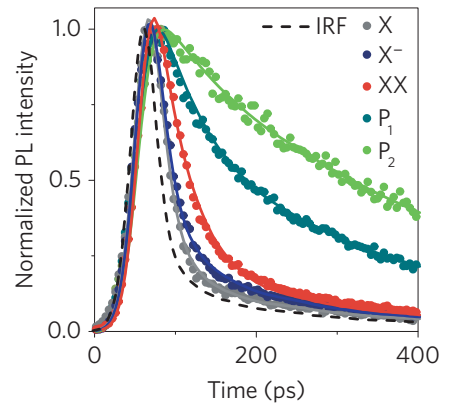

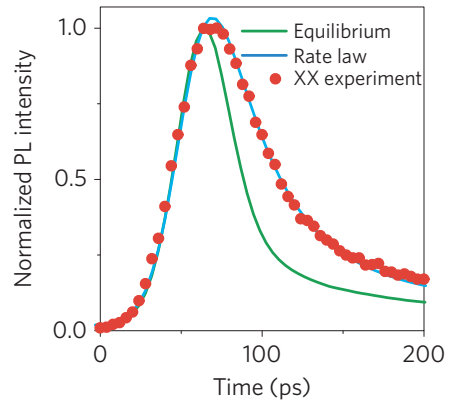

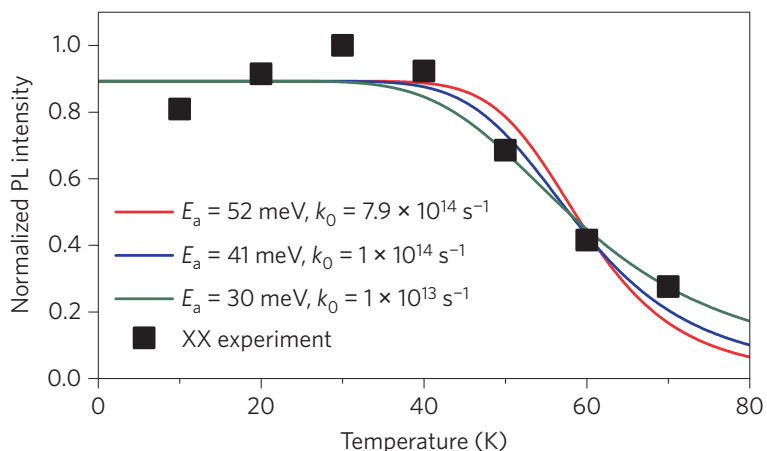

Figure 2 | Dynamics and thermal stability of excited states. a, Time-resolved $P L$ traces for $X, X^{-}, X X, P_{1}$ and $P_{2}$ species (dots, with solid lines as guides to the eye) and the instrument response function (IRF). The two defect-related peaks, $P_{1}$ and $P_{2}$, have decay times $>100 p s$, whereas the $X, X^{-}$and $X X$ features decay rapidly. Based on a single exponential fit, the emission lifetimes are $\tau_{X}=14 \mathrm{ps}, \tau_{X^{-}}=25 \mathrm{ps}$ and $\tau_{\mathrm{XX}}=31 \mathrm{ps}$, with uncertainties of $\pm 5 \mathrm{ps}$. b, Experimental TR-PL for the biexciton species compared with the expected behaviour based on the measured exciton dynamics. The green line is a fit based on an equilibrium model with a quadratic relation between the biexciton and exciton populations. The blue line is based on the rate equation described in the text. c, The squares show the relative PL emission measured from the biexciton state as a function of sample temperature for an applied laser fluence of $0.8 \mu \mathrm{J} \mathrm{cm}^{-2}$. The solid curves are predicted emission strengths based on the thermal dissociation model described in the text for the indicated activation energies $E_{\mathrm{a}}$ and pre-factors $k_{0}$.

relation between the density of biexcitons and excitons ${ }^{17}$ - that is, $I_{\mathrm{XX}} \propto I_{\mathrm{X}}{ }^{\alpha}$ with $\alpha=2$. However, in quantum-well systems, for which biexcitons have been extensively studied ${ }^{7}$, exponents of $\alpha=1.2-1.9$ are typically observed, and attributed to the lack of equilibrium between the states ${ }^{18,26}$. The strong biexciton emission compared to exciton emission that we observe has previously been reported in quantum-well systems at low temperatures and elevated excitation densities ${ }^{27}$.

To further examine the biexciton state, we have investigated its dynamics using time-resolved PL (TR-PL). In Fig. 2a, we compare TR-PL traces for XX emission with those from the excitonic states $\left(\mathrm{X}, \mathrm{X}^{-}\right)$and from the strongest defect species $\left(\mathrm{P}_{1}, \mathrm{P}_{2}\right)$. All of the time traces can be fitted to a rapid rise, comparable to the instrumental response, but the decay times differ strongly. The lifetimes of the exciton, trion and biexciton states are tens of picoseconds, whereas the emission lifetime for $\mathrm{P}_{1}$ and $\mathrm{P}_{2}$ defect features exceeds $100 \mathrm{ps}$. This result provides further evidence for the validity of the assignment of the $\mathrm{P}_{0}$ emission as arising from biexcitons, rather than from any type of defect state. The short emission times of $\mathrm{X}, \mathrm{X}^{-}$and $\mathrm{XX}$ features are compatible with the lack of full thermal equilibrium between the exciton and biexciton, as mentioned above. The emission times of the exciton and biexciton states were investigated as a function of the pump fluence. No appreciable variation in the emission time with fluence was observed. The result indicates that depletion of excitons by an exciton-exciton annihilation process ${ }^{28,29}$ is not significant under our experimental conditions.

We can use the inferred single exciton dynamics to predict the biexciton dynamics and compare with the observed biexciton TRPL. Under the assumption of full thermal equilibrium, the biexciton density $N_{\mathrm{Xx}}$ will vary quadratically with the exciton density $N_{\mathrm{X}}$ at all times $^{17}$. From the measured dynamics for $N_{X}$, we then predict the TR-PL for biexciton emission (Fig. 2b), which clearly does not match experimental results. On the other hand, the measured biexciton 
a

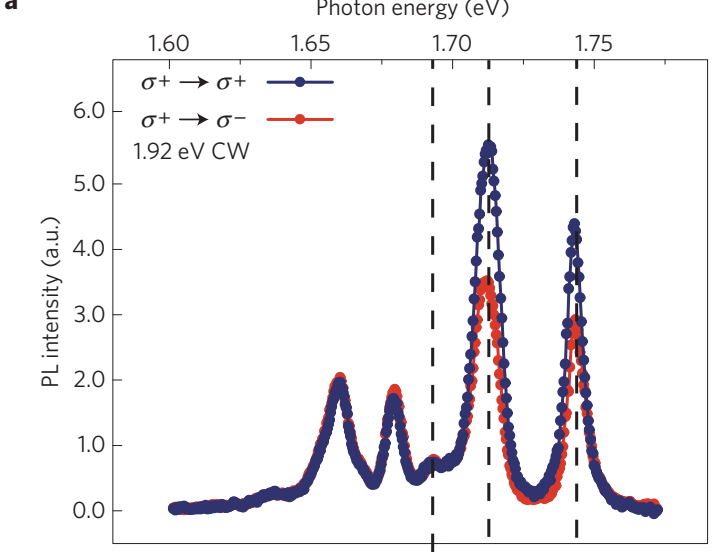

b

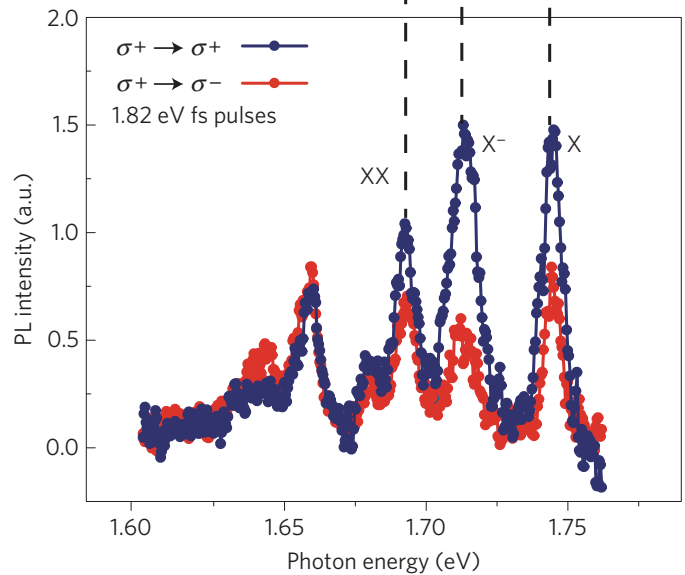

Figure 3 | Analysis of circularly polarized emission components for excitation by near-resonant circularly polarized radiation. $\mathbf{a}, \mathbf{b}, \mathrm{PL}$ spectra for the same (blue) and opposite (red) circularly polarized states for the $\mathrm{WSe}_{2}$ monolayer at $15 \mathrm{~K}$. The emission energies for neutral $(X)$ and charged $\left(X^{-}\right)$excitons and the biexciton $(X X)$ state are indicated by dashed lines.

a, Results for low exciton density with $\mathrm{CW}$ excitation at a photon energy of $1.92 \mathrm{eV}$. Defining the degree of circular polarization as

$\rho=\left[I\left(\sigma^{+}\right)-I\left(\sigma^{-}\right)\right] /\left[I\left(\sigma^{+}\right)+I\left(\sigma^{-}\right)\right]$, where $\sigma^{ \pm}$denotes the polarization state detected for $\sigma^{+}$excitation, we obtain $\rho(X)=0.20$ and $\rho\left(X^{-}\right)=0.38$, whereas $|\rho|<0.05$ for the defect states. $\mathbf{b}$, Results for high exciton density with pulsed excitation at a photon energy of $1.82 \mathrm{eV}$. The biexciton feature is now present in the PL spectra and exhibits significant circular polarization, with $\rho(X X)=0.16$. The other features show similar behaviour as for the case of low excitation density, with $\rho(X)=0.30$ and $\rho\left(X^{-}\right)=0.50$, whereas $|\rho|<0.05$ for the defect states.

TR-PL is compatible with predictions (Fig. 2b) of a non-thermalized model based on a simple rate-equation:

$$
\frac{\mathrm{d} N_{\mathrm{Xx}}}{\mathrm{d} t}=\beta N_{\mathrm{X}}^{2}-\gamma N_{\mathrm{XX}}
$$

Here $\beta=1.1 \mathrm{~cm}^{2} \mathrm{~s}^{-1}$ represents the biexciton formation rate from collisions of excitons, where we have assumed in the analysis that each absorbed photon initially produces an exciton. Using kinetic theory for thermalized excitons, we infer from $\beta$ (Methods) a crosssection for biexciton formation of $\sim 4 \mathrm{~nm}$, comparable to the exciton Bohr radius ${ }^{30}$. Our fitting procedure yields a biexciton relaxation time of $\gamma^{-1}=27 \mathrm{ps}$, which is attributed primarily to non-radiative decay channels. The biexciton dissociation process is suppressed at low temperatures, as discussed below. This effectively decouples the exciton and biexciton dynamics, permitting a longer lifetime for biexcitons than for excitons. A similar experimental finding of a

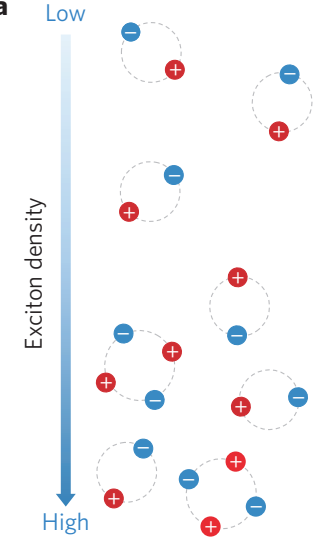

b

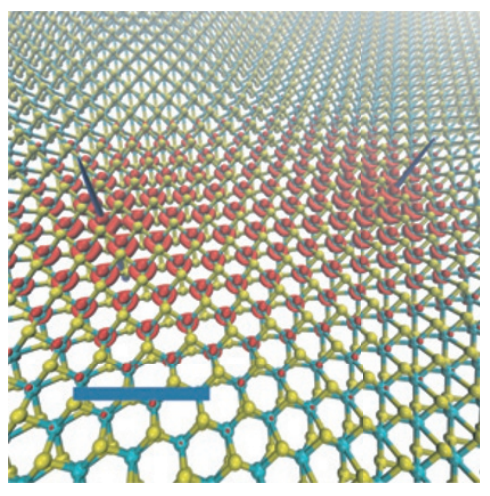

Figure 4 | Real-space representation of biexcitons. a, Schematic representation of biexcitons as four-body quasiparticles. With increasing exciton density biexcitons are formed from excitons. $\mathbf{b}$, Real-space representation of the biexciton projected onto the $\mathrm{WS}_{2}$ plane as determined by the variational calculation described in the text. The red regions indicate distribution of the total charge of the two electrons in the biexciton when the two holes (blue peaks) are fixed at a typical separation of $3.3 \mathrm{~nm}$. The scale bar is $1 \mathrm{~nm}$.

longer biexciton than exciton lifetimes has been previously reported in the literature for ( $\mathrm{Zn}, \mathrm{Cd}) \mathrm{Se} / \mathrm{ZnSe}$ quantum wells ${ }^{27}$.

The nature of biexcitonic states and the valley occupancy of these states can be probed through investigation of the polarization characteristics of the photoluminescence. To this end, we used near-resonant circularly polarized excitation (Methods) to produce excitons preferentially in one valley ( $\mathrm{K}$ or $\mathrm{K}^{\prime}$; refs 9-12). For low exciton density under continuous wave (CW) laser excitation, the $\mathrm{X}, \mathrm{X}^{-}$and defect-related emission peaks are observed (Fig. 3a); at high exciton density produced by pulsed laser excitation, we also observe XX emission (Fig. 3b). The peaks for X, $\mathrm{X}^{-}$and XX (under pulsed excitation) all exhibit significant circular polarization. The two major defect-related emission peaks, on the other hand, show no measurable circular polarization character. This result provides additional evidence that defects do not play a role in the peak identified as the biexciton.

The polarization of the biexciton emission also reflects the valley character of this many-body state. Exchange and correlation effects could induce differences in the stability of biexcitons formed from two excitons in the same valley or in opposite valleys. For near-resonant excitation with circularly polarized light, we observe biexciton emission with both the same and opposite circularly polarized state (Fig. 3b). We expect the former to arise more strongly from intravalley excitons than the latter. The biexciton emission spectra for these two cases, as well as for linearly polarized excitation and excitation with higher photon energy, are indistinguishable. The measurements thus suggest that both intra- and intervalley biexcitons can be formed and that they exhibit the same emission energies (within $1 \mathrm{meV}$ ).

Taken together, the observed fluence dependence, temporal dynamics and circular polarization properties of the $\mathrm{P}_{0}$ feature provide strong evidence for its assignment as a biexciton feature. We now consider experimental observations related to the biexciton binding energy and thermal stability.

The biexciton binding energy is defined as the difference in energy between two free excitons and the biexciton state: $\Delta_{\mathrm{XX}}=2 E_{\mathrm{X}}-E_{\mathrm{XX}}$. We determine $E_{\mathrm{XX}}$ through observation of the biexciton photoluminescence at energy $\hbar \omega_{\mathrm{xx}}$ and information about the remaining excitation in the material. If we assume that radiative decay of the biexciton produces an exciton, then 
$E_{\mathrm{xx}}=\hbar \omega_{\mathrm{xx}}+E_{\mathrm{x}}=\hbar \omega_{\mathrm{xx}}+\hbar \omega_{\mathrm{x}}$, where $\hbar \omega_{\mathrm{x}}$ denotes the exciton emission energy. Thus, the biexciton binding energy is given by the spectral shift: $\Delta_{\mathrm{Xx}}=\hbar \omega_{\mathrm{x}}-\hbar \omega_{\mathrm{Xx}}=52 \mathrm{meV}$. We note that in this system there are other types of excitons-namely, trions and dark excitons ${ }^{31}$. We discuss the possible influence of these species in the Supplementary Information. We also present experimental data on the change in emission energies and intensities induced by different dielectric environments and charge densities of the sample.

We investigated the stability of the biexciton using temperaturedependent PL measurements. Under the same excitation condition, the biexciton emission intensity shows a plateau at low temperature, but decreases significantly for temperature $T>70 \mathrm{~K}$ (Fig. 2c). This trend can be explained by a model in which biexcitons have a roughly constant rate of formation as a function of $T$, but exhibit a thermally activated dissociation channel, corresponding to a decay rate in equation (1) of the form $\gamma(T)=\tau_{\mathrm{xx}}{ }^{-1}+k_{0} \exp \left(-E_{\mathrm{a}} / k_{\mathrm{B}} T\right)$. The observed temperature dependence is compatible with activation energies $E_{\mathrm{a}}$ from 30 to $50 \mathrm{meV}$ (see Fig. $2 \mathrm{c}$ and Supplementary Section 7).

It is instructive to compare the biexciton binding energy in $\mathrm{WSe}_{2}$ monolayers with those of conventional quasi-2D structures. The value of $\Delta_{\mathrm{xx}}=52 \mathrm{meV}$ for the former exceeds that found in III-V quantum wells by almost two orders of magnitude ${ }^{7}$. This remarkably large $\Delta_{\mathrm{Xx}}$ must, however, be considered in light of the overall strength of the Coulomb interactions. If we normalize $\Delta_{\mathrm{xx}}$ in WSe to the exciton binding energy in the same system, recently reported as $370 \mathrm{meV}$ (ref. 5), we obtain a ratio, the so-called Haynes factor, of 0.14 . This ratio is similar to that found in quantum-well systems. The large $\Delta_{\mathrm{Xx}}$ in monolayer $\mathrm{WSe}_{2}$ thus scales with the overall strength of the Coulomb interaction.

To obtain deeper physical insight into the biexciton state, we have performed quantum-mechanical calculations of these correlated four-particle states, represented schematically in Fig. 4a. Although biexciton states have been previously investigated for quasi-2D systems $s^{32-34}$, the results cannot be directly applied to the TMDC monolayers because of the distinctive character of the electronhole $(\mathrm{e}-\mathrm{h})$ interaction in these atomically thin materials. As has been discussed in the recent theoretical literature $e^{30,35}$ and revealed in the spectrum of the excited exciton states ${ }^{3-5}$, the strongly inhomogeneous dielectric environment gives rise to a non-local screening effect for the e-h interaction potential: the screening is strong at short range, but weak at long range. Using a biexciton Hamiltonian with such an $\mathrm{e}-\mathrm{h}$ interaction potential, we have carried out a variational calculation that yields a biexciton binding energy of $37 \mathrm{meV}$ for $\mathrm{WSe}_{2}$ (see Methods and Supplementary Section 8). On the basis of a comparison with existing numerically exact results for a conventional screened Coulomb potential ${ }^{36}$, we expect that the true biexciton binding energy should exceed our variational bound by an additional $40-50 \%$. This yields a predicted $\Delta_{\mathrm{Xx}} \sim 50-60 \mathrm{meV}$, in good agreement with experiment.

The nature of the biexciton states in $\mathrm{WSe}_{2}$ monolayers is elucidated by examining the real-space structure of the biexciton complex predicted by our variational calculation. The biexciton consists of two distinct excitons, each with a Bohr radius equal to that of a single exciton $(1 \mathrm{~nm})$, separated by a distance three to four times larger (Fig. 4b). Given the large separation between charges, the screening of the Coulomb interaction will be strongly influenced by that of the external media, rather than the intrinsic screening of the $\mathrm{WSe}_{2}$ monolayer. We thus expect the biexciton states to be particularly sensitive to the nature of the surrounding media.

The results presented here demonstrate that under appropriate conditions the strongest channel for light emission can occur through a biexciton channel. The prominence of such four-body correlated states in the material reflects the unusual strength of many-body interactions in atomically thin TMDC semiconductors. Monolayer TMDC materials are thus very favourable candidates for other many-body processes, such as multiple-exciton generation ${ }^{37}$, and for the creation of new higher-order correlated states, such as exciton condensates or the recently introduced dropletons ${ }^{8}$. The existence of the degenerate $\mathrm{K}$ and $\mathrm{K}^{\prime}$ valleys also offers the possibility of creating optically bright biexciton states with pairs of carriers in distinct valleys. This not only induces new types of quantum coherent excitation within the solid, but also offers the possibility of the creation, through cascaded emission, of correlated photon pairs with a novel mechanism for the control of polarization states ${ }^{38}$.

\section{Methods}

Methods and any associated references are available in the online version of the paper.

\section{Received 2 October 2014; accepted 24 March 2015;} published online 11 May 2015

\section{References}

1. Mak, K. F., Lee, C., Hone, J., Shan, J. \& Heinz, T. F. Atomically thin MoS: A new direct-gap semiconductor. Phys. Rev. Lett. 105, 136805 (2010).

2. Splendiani, A. et al. Emerging photoluminescence in monolayer $\mathrm{MoS}_{2}$. Nano Lett. 10, 1271-1275 (2010)

3. Chernikov, A. et al. Exciton binding energy and nonhydrogenic Rydberg series in monolayer WS . Phys. Rev. Lett. 113, 076802 (2014).

4. Ye, Z. et al. Probing excitonic dark states in single-layer tungsten disulphide. Nature 513, 214-218 (2014).

5. He, K. et al. Tightly bound excitons in monolayer WSe $\mathrm{W}_{2}$. Phys. Rev. Lett. 113, 026803 (2014)

6. Ugeda, M. M. et al. Giant bandgap renormalization and excitonic effects in a monolayer transition metal dichalcogenide semiconductor. Nature Mater. 13, 1091-1095 (2014).

7. Klingshirn, C. F. Semiconductor Optics Vol. 3 (Springer, 2007)

8. Almand-Hunter, A. E. et al. Quantum droplets of electrons and holes. Nature 506, 471-475 (2014)

9. Mak, K. F., He, K., Shan, J. \& Heinz, T. F. Control of valley polarization in monolayer $\mathrm{MoS}_{2}$ by optical helicity. Nature Nanotech. 7, 494-498 (2012).

10. Zeng, H., Dai, J., Yao, W., Xiao, D. \& Cui, X. Valley polarization in $\mathrm{MoS}_{2}$ monolayers by optical pumping. Nature Nanotech. 7, 490-493 (2012).

11. Cao, T. et al. Valley-selective circular dichroism of monolayer molybdenum disulphide. Nature Commun. 3, 887 (2012).

12. Jones, A. M. et al. Optical generation of excitonic valley coherence in monolayer $\mathrm{WSe}_{2}$. Nature Nanotech. 8, 634-638 (2013).

13. Mak, K. F., McGill, K. L., Park, J. \& McEuen, P. L. Valleytronics. The valley Hall effect in $\operatorname{MoS}(2)$ transistors. Science 344, 1489-1492 (2014).

14. Mak, K. F. et al. Tightly bound trions in monolayer $\mathrm{MoS}_{2}$. Nature Mater. 12, 207-211 (2013)

15. Ross, J. S. et al. Electrical control of neutral and charged excitons in a monolayer semiconductor. Nature Commun. 4, 1474 (2013).

16. Mitioglu, A. et al. Optical manipulation of the exciton charge state in single-layer tungsten disulfide. Phys. Rev. B 88, 245403 (2013).

17. Kim, J., Wake, D. \& Wolfe, J. Thermodynamics of biexcitons in a GaAs quantum well. Phys. Rev. B 50, 15099-15107 (1994).

18. Phillips, R., Lovering, D., Denton, G. \& Smith, G. Biexciton creation and recombination in a GaAs quantum well. Phys. Rev. B 45, 4308-4311 (1992).

19. Colombier, L. et al. Detection of a biexciton in semiconducting carbon nanotubes using nonlinear optical spectroscopy. Phys. Rev. Lett. 109, 197402 (2012)

20. Yuma, B. et al. Biexciton, single carrier, and trion generation dynamics in single-walled carbon nanotubes. Phys. Rev. B 87, 205412 (2013).

21. Klimov, V. I. Semiconductor and Metal Nanocrystals: Synthesis and Electronic and Optical Properties (CRC Press, 2003).

22. Mai, C. et al. Many-body effects in valleytronics: Direct measurement of valley lifetimes in single-layer $\mathrm{MoS}_{2}$. Nano Lett. 14, 202-206 (2014).

23. Shang, J. et al. Observation of excitonic fine structure in a $2 \mathrm{D}$ transition-metal dichalcogenide semiconductor. ACS Nano 9, 647-655 (2015).

24. Tongay, S. et al. Defects activated photoluminescence in two-dimensional semiconductors: Interplay between bound, charged, and free excitons. Sci. Rep. 3, 02657 (2013)

25. Wang, G. et al. Valley dynamics probed through charged and neutral exciton emission in monolayer WSe $\mathrm{W}_{2}$. Phys. Rev. B 90, 075413 (2014).

26. Birkedal, D., Singh, J., Lyssenko, V. G., Erland, J. \& Hvam, J. M. Binding of quasi-two-dimensional biexcitons. Phys. Rev. Lett. 76, 672-675 (1996)

27. Spiegel, R. et al. Polarization-dependent formation of biexcitons in (Zn,Cd)Se/ZnSe quantum wells. Phys. Rev. B 55, 9866-9871 (1997). 
28. Kumar, N. et al. Exciton-exciton annihilation in $\mathrm{MoSe}_{2}$ monolayers. Phys. Rev. B 89, 125427 (2014).

29. Sun, D. et al. Observation of rapid exciton-exciton annihilation in monolayer molybdenum disulfide. Nano Lett. 14, 5625-5629 (2014).

30. Berkelbach, T. C., Hybertsen, M. S. \& Reichman, D. R. Theory of neutral and charged excitons in monolayer transition metal dichalcogenides. Phys. Rev. B 88, 045318 (2013).

31. Liu, G-B., Shan, W-Y., Yao, Y., Yao, W. \& Xiao, D. Three-band tight-binding model for monolayers of group-VIB transition metal dichalcogenides. Phys. Rev. B 88, 085433 (2013).

32. Kleinman, D. Binding energy of biexcitons and bound excitons in quantum wells. Phys. Rev. B 28, 871-879 (1983).

33. Singh, J., Birkedal, D., Lyssenko, V. G. \& Hvam, J. M. Binding energy of two-dimensional biexcitons. Phys. Rev. B 53, 15909-15913 (1996).

34. Riva, C., Peeters, F. M. \& Varga, K. Excitons and charged excitons in semiconductor quantum wells. Phys. Rev. B 61, 13873-13881 (2000).

35. Cudazzo, P., Tokatly, I. V. \& Rubio, A. Dielectric screening in two-dimensional insulators: Implications for excitonic and impurity states in graphane. Phys. Rev. B 84, 085406 (2011).

36. Usukura, J., Suzuki, Y. \& Varga, K. Stability of two- and three-dimensional excitonic complexes. Phys. Rev. B 59, 5652-5661 (1999).

37. Choi, Y., Sim, S., Lim, S. C., Lee, Y. H. \& Choi, H. Ultrafast biexciton spectroscopy in semiconductor quantum dots: Evidence for early emergence of multiple-exciton generation. Sci. Rep. 3, 3206 (2013)

38. Akopian, N. et al. Entangled photon pairs from semiconductor quantum dots. Phys. Rev. Lett. 96, 130501 (2006).

\section{Acknowledgements}

The authors would like to acknowledge valuable discussions with A. Chernikov and T. Cao and technical assistance from Y. Rao and F. Zhang. The experimental research was supported by the National Science Foundation through grants DMR-1106172 and DMR-1122594, the Keck Foundation, and the Honda Research Institute. Support for data analysis by was provided by the AMOS program, Chemical Sciences, Geosciences, and Biosciences Division, Basic Energy Sciences, US Department of Energy under Contract No. DE-AC02-76-SFO0515 (T.F.H.). This work was carried out in part at the Center for Functional Nanomaterials, Brookhaven National Laboratory, which is supported by the US Department of Energy, Office of Basic Energy Sciences, under Contract No. DE-AC02-98CH10886 (M.S.H.)

\section{Author contributions}

Y.Y. and X-X.Z. designed the experiment, performed the measurements, interpreted the results, and wrote the manuscript; T.C.B. performed the variational calculation under the guidance of M.S.H. and D.R.R. and contributed to writing of the manuscript; T.F.H. contributed to the interpretation of the results and writing of the manuscript.

\section{Additional information}

Supplementary information is available in the online version of the paper. Reprints and permissions information is available online at www.nature.com/reprints. Correspondence and requests for materials should be addressed to T.F.H

\section{Competing financial interests}

The authors declare no competing financial interests. 


\section{Methods}

Experimental. Monolayer WSe ${ }_{2}$ crystals were prepared by mechanical exfoliation on a Si substrate covered by an oxide layer and exhibited unintentional n-doping ${ }^{12}$ (see Supplementary Information for further information on sample preparation and other experimental details). The samples were studied in an optical cryostat using an inverted microscope. The excitation source for the photoluminescence measurements consisted of femtosecond laser pulses with a photon energy of $3.06 \mathrm{eV}$ for the fluence-dependence study and time-resolved PL. These pulses, of $100 \mathrm{fs}$ duration, were produced by the frequency-doubled output of a mode-locked Ti:sapphire laser operating at $80 \mathrm{MHz}$. For circular polarization measurements, femtosecond excitation pulses with a photon energy centred at $1.82 \mathrm{eV}$ and a $1 \mathrm{MHz}$ repetition rate were used. We obtained these pulses by filtering the supercontinuum radiation produced by focusing an amplified mode-locked fibre laser (Impulse, Clarke-MXR) in an undoped YAG crystal. Continuous radiation at $1.92 \mathrm{eV}$ for the comparison study was provided by a solid-state laser.

For the $3.06 \mathrm{eV}$ excitation, we computed the applied laser fluence on the sample using the measured spot diameter of $1.4 \mu \mathrm{m}$ produced by a $\times 40$ microscope objective. Taking into account the influence of the substrate and the dielectric function of the $\mathrm{WSe}_{2}$ monolayer ${ }^{39}$, we find that the absorbed fluence is $2.2 \%$ of the applied fluence. An upper bound for the exciton density is obtained by assuming full conversion of the absorbed photons into excitons. This is probably an overestimate of the true exciton density, as recent reports indicate incomplete relaxation of carriers produced at higher photon energies to band-edge excitons ${ }^{40}$. The pump laser radiation, in addition to producing excitons, can potentially change the nature of the sample by altering the charge density (through photodoping) or the temperature (through heating effects). Although some photodoping was observed, the degree of photodoping was essentially independent of laser fluence (Supplementary Information). Hence, this process does not influence the measured fluence-dependent properties. Similarly, equilibrium heating was shown to be negligible based on the lack of spectral shifts (see Supplementary Information).

We measured the emitted PL in a back-scattering configuration with a grating spectrometer and a liquid-nitrogen cooled CCD for spectroscopic analysis. As an alternative, we detected the PL with a fast avalanche photodiode (PicoQuant PDM) and analysed the temporal profile of the emission by time-resolved single photon counting (PicoQuant PicoHarp 300). We determined the instrument response function (IRF) of the TR-PL set-up using $1.53 \mathrm{eV}$ fs pulses from the Ti:sapphire laser. In comparing models with the experimental time traces, we convolved the predicted form with the measured IRF.

Theoretical. Within the rate-equation model of the dynamics, the cross-section $\sigma$ for biexciton formation is estimated from the parameter $\beta$ in equation (1) using the kinetic theory relation $\beta=\sigma v_{\text {rel }}$, where $v_{\text {rel }}$ is the relative exciton velocity. Assuming a 2D Maxwellian velocity distribution at $10 \mathrm{~K}$ for excitons of mass $0.68 \mathrm{~m}_{\mathrm{e}}$ (refs 3,30), we infer a biexciton formation cross-section of $\sigma \approx 4 \mathrm{~nm}$. As the actual exciton velocity distribution may exceed that implied by the base temperature of the sample (because of hot carrier effects), this analysis may overestimate the formation cross-section. Still, the inferred value is comparable to the exciton Bohr radius of $\sim 1 \mathrm{~nm}$ (ref. 30).

We examined the biexciton states in WSe $\mathrm{W}_{2}$ monolayers theoretically using a variational calculation within an effective mass approximation to describe carriers at the $\mathrm{K} / \mathrm{K}^{\prime}$ band edge (see Supplementary Information). Although the general scheme is similar to that applied in previous studies of quantum wells, a critical difference is the form of the Coulomb interaction between charges. Because of the atomic-level thickness of the material, the dielectric screening is non-local in character. We model the interaction using the electrostatic interaction for charges in a 2D dielectric sheet. The e-h potential in reciprocal space is then given by ${ }^{30,35} V(q)=-2 \pi e^{2} / q\left(1+r_{0} q\right)$, where $r_{0}$ is a screening length, which is proportional to the $2 \mathrm{D}$ polarizability of the sheet. For the WSe $\mathrm{W}_{2}$ monolayer, we use $r_{0}=45 \AA$ and electron and hole mass parameters of $m_{\mathrm{e}}=m_{\mathrm{h}}=0.34 m_{0}$, where $m_{0}$ denotes the free electron mass $^{3,30}$. The variational wavefunction, which includes six variational parameters, allows interpolation of the biexciton configuration from equal sharing of charges to two completely separated excitons, as well as inclusion of the correlation between like charges. Both pairs of electrons and holes are assumed to be in singlet states. (See Supplementary Section 8, for details concerning the calculation.)

\section{References}

39. Li, Y. et al. Measurement of the optical dielectric function of monolayer transition-metal dichalcogenides: $\mathrm{MoS}_{2}, \mathrm{MoSe}_{2}, \mathrm{WS}_{2}$, and WSe $\mathrm{W}_{2}$. Phys. Rev. B 90, 205422 (2014)

40. Kozawa, D. et al. Photocarrier relaxation pathway in two-dimensional semiconducting transition metal dichalcogenides. Nature Commun. 5, 4543 (2014) 